\title{
Surgical treatment of ventricular tachycardia after epicardial mapping studies
}

\author{
R. A. J. Spurrell, ${ }^{1}$ A. K. Yates, C. W. Thorburn, G. E. Sowton, and D. C. Deuchar \\ From The Departments of Cardiology and Cardiothoracic Surgery, Guy's Hospital, London
}

Two patients with intractable life-threatening ventricular tachycardias have been studied using intracardiac electrograms and programmed electrical stimulation of the heart. Both patients were shown to have an underlying re-entry mechanism in the ventricles as the basis for the tachycardias. Both patients underwent epicardial mapping studies at cardiac surgery, and the site of re-entry was established. In one patient the re-entry front was found to start in the posterobasal region of the left ventricle and in the other patient the re-entry front was found in the anterobasal region of the right ventricle. In both patients surgical interruption of the re-entry front was carried out. Both patients are alive and free from tachycardias at the time of writing.

In recent years much discussion has centred around the underlying mechanisms of ventricular tachycardia. The two main mechanisms postulated are that the tachycardia is due to a rapidly discharging focus or that some form of re-entry mechanism is responsible. Lown, Kleiger, and Williams (1965) showed that ventricular tachycardia could develop in the dog's heart exposed to large amounts of digitalis and suggested that this was because of the development of an increased frequency of discharge from the cardiac cells. Recently Wellens, Schuilenburg, and Durrer (1972) demonstrated in 5 patients with ventricular tachycardia that a reciprocal mechanism was the most probable underlying mechanism though the precise site of re-entry was uncertain. Spurrell, Sowton, and Deuchar (1973) using similar techniques showed that a reentry or reciprocal mechanism in the main intraventricular bundle-branches was the most probable basis for ventricular tachycardia in 4 patients. This paper describes 2 patients with intractable ventricular tachycardia in whom investigations by programmed cardiac electrical stimulation in conjunction with intracardiac recordings indicated a reentry mechanism. Both patients underwent surgery and the re-entry process was then further studied using epicardial mapping. From the results obtained from the intraoperative mapping studies a surgical approach specific to each patient was de-

Received Io June I974.

1 Cardiac Department, St. Bartholomew's Hospital, London ECI. vised to interrupt the re-entry circuit and prevent further attacks of tachycardia.

\section{Patients and methods}

The full details of the 2 patients are given under individual case histories. The basic clinical details are summarized in the Table.

Case $I$ suffered an inferior myocardial infarction on 13 December 1972. He began to develop attacks of ventricular tachycardia for which DC cardioversion was required on 7 January 1973 . He had 9 subsequent admissions to the local hospital with ventricular tachycardia requiring DC cardioversion, and was then referred to the Brompton Hospital, London, on ro August 1973, where he had a temporary right ventricular pacing electrode inserted. Over the next few days he had I9 attacks of tachycardia terminated either by overdrive ventricular pacing or by DC cardioversion, and on one occasion DC cardioversion was required to terminate ventricular fibrillation. Cardiac catheterization and angiocardiography at this time showed complete occlusion of the posterior descending branch of the right coronary artery and a 50 per cent stenosis in the left anterior descending coronary artery. A left ventriculogram demonstrated an akinetic area in the region of the inferior wall of the left ventricle but no frank aneurysm. On 21 September 1973 he was transferred to Guy's Hospital, where he had a further 16 attacks of tachycardia and 2 attacks of ventricular fibrillation over a period of 8 days. During this period an electrophysiological study was carried out and after this he underwent surgery for interruption of a re-entry pathway in his left ventricle on 28 September 1973. After this it was not possible to reinitiate the tachycardia with suitable premature beats but in the two months after operation he 
TABLE Clinical details

\begin{tabular}{|c|c|c|c|c|c|}
\hline $\begin{array}{l}\text { Case } \\
\text { No. }\end{array}$ & $\begin{array}{l}\text { Age } \\
(y r)\end{array}$ & Sex & Aetiology of tachycardia & Drugs & $\begin{array}{l}\text { Surface electrocardiogram } \\
\text { during tachycardia }\end{array}$ \\
\hline $\mathbf{I}$ & 57 & $\mathbf{M}$ & Coronary artery disease & $\begin{array}{l}\text { Quinidine; } \\
\text { lignocaine; } \\
\text { procainamide; } \\
\text { diphenylhydantoin; } \\
\text { practolol }\end{array}$ & $\begin{array}{l}\text { Ventricular tachycardia; } \\
\text { right bundle-branch block; } \\
\text { frontal plane QRS axis }+125^{\circ} \text {; } \\
\text { rate } 165 / \mathrm{min}\end{array}$ \\
\hline 2 & 35 & $M$ & ? Cardiomyopathy & $\begin{array}{l}\text { Lignocaine; } \\
\text { procainamide; } \\
\text { practolol; } \\
\text { propranolol; } \\
\text { diphenylhydantoin }\end{array}$ & $\begin{array}{l}\text { Ventricular tachycardia; } \\
\text { left bundle-branch block; } \\
\text { frontal plane QRS axis }-30^{\circ} \text {; } \\
\text { rate } 205 / \mathrm{min} \text {; } \\
\text { on } 4 \text { occasions rate } 270 / \mathrm{min}\end{array}$ \\
\hline
\end{tabular}

had a total of 3 further attacks of ventricular tachycardia. Since then he has had no further attacks for 5 months at the time of writing.

Case 2 experienced a sudden loss of senses while fishing on 18 April I966. He was admitted to hospital and found to be in ventricular tachycardia; this attack terminated spontaneously. On 9 March 1967 he was admitted to Guy's Hospital after a dizzy spell and was again found to be in ventricular tachycardia. He lost consciousness on admission to hospital and required DC cardioversion. He was treated with propranolol and had only $\mathrm{I}$ to 2 attacks of rapid, regular palpitation each year, which terminated spontaneously. In 1972 the attacks began to occur more frequently, up to I to 2 each fortnight, and during the attacks he felt faint. Resting electrocardiograms at this time showed persistent $T$ wave inversion in leads III and VI to V4. On 2 December I973 he was again admitted to Guy's Hospital with a further attack of ventricular tachycardia which was terminated by DC cardioversion. Over the next 3 weeks he had 8 further attacks of tachycardia. Drug therapy was found to be ineffective, and though the tachycardia could be interrupted by DC cardioversion it restarted within seconds; a right ventricular pacing electrode was inserted and the attacks were terminated using suitably timed right ventricular premature beats. An electrophysiological study was carried out and after this on I5 January 1974 he underwent surgery for interruption of a re-entry pathway in his right ventricle. After this it was not possible to reinitiate a tachycardia using ventricular premature beats and he is now well and has had no attacks of tachycardia 4 months after operation at the time of writing.

The preoperative electrophysiological studies were performed using three bipolar electrode catheters introduced percutaneously through one or both femoral veins. Two of the catheters were positioned in the right atrium, one for recording the right atrial electrogram and one for pacing the right atrium; this latter catheter could also be positioned in the right ventricle for ventricular pacing and inducing right ventricular premature beats. The third catheter was positioned across the tricuspid valve and the His bundle electrogram re- corded using the technique described by Scherlag et al. (I969).

All recordings were made on an 8-channel Elema Mingograf $8 \mathrm{r}$. The His bundle electrogram was recorded with the frequency response set at 45 to 500 cycles/s. Leads I, III, VI, and V6 of the surface electrocardiogram were recorded simultaneously with the intracardiac recordings.

A Devices ${ }^{1} 4270$ stimulator was used for atrial and ventricular pacing. The heart was driven at a constant frequency and one or more atrial or ventricular premature beats were delivered after every eighth beat of the basic driven rhythm. A variable delay circuit triggered by the $\mathbf{R}$ wave of the surface electrocardiogram was used so that the premature beats could be delivered in the cardiac cycle at a preset delay after the preceding driven beat. In each patient the mode of initiation and termination of the tachycardia and the effect of ventricular premature beats on the basic rhythm during tachycardia were studied.

The criteria used for the diagnosis of a reciprocal mechanism, rather than a protected ectopic focus, in patients with tachycardia are that the tachycardia can be initiated by suitably timed premature beats, interpolated premature beats induced during tachycardia are followed by pauses less than compensatory, and the tachycardia can be terminated by suitably timed premature beats (Wellens et al., 1972).

\section{Operative physiological study}

After exposure of the heart at thoracotomy unipolar pacing wires were sutured to the apex of the left ventricle. The reference electrode was a unipolar electrode probe with a $2 \mathrm{~mm}$ electrode surface which was inserted via a stab incision through the apex of the left ventricle into the left ventricular cavity in Case $I$ and into the right ventricular cavity in Case 2. The indifferent electrode was attached to the fat in the region of the aortic root. Bipolar epicardial complexes were obtained using a hand held electrode with a $2 \mathrm{~mm}$ interelectrode spacing, each electrode being $0.5 \mathrm{~mm}$ in diameter.

The unipolar signals from the left ventricular cavity

${ }^{1}$ Devices Instruments Ltd., Welwyn Garden City, Herts. 
and the bipolar epicardial signals were fed into the isolation unit of an Elema Mingograf $8 \mathrm{r}$ and were recorded with the frequency response set at 35 to $400 \mathrm{~Hz}$.

All recordings were made simultaneously with leads I, II, and III of the surface electrocardiogram at paper speeds of 100 and $1000 \mathrm{~mm} / \mathrm{s}$. Before these recordings, a drawing was made of the left ventricle in Case $I$ and of the right ventricle in Case $2 ; 40$ to 50 selected points were chosen and marked on the drawing. During sinus rhythm epicardial recordings were obtained from these preselected points. The ventricular tachycardia was then initiated by suitably timed ventricular premature beats (as determined at the pre-operative study) and again epicardial recordings were obtained from the preselected sites during ventricular tachycardia.

Epicardial activation times were obtained using the onset of the left or right ventricular cavity potential as the reference. Measurements were made from the onset of the ventricular cavity potential to the intrinsic deflection of the epicardial potential.

From the results obtained, maps of the epicardial activation sequence were constructed by connecting points of equal activation times at $5 \mathrm{~ms}$ intervals. In this way the epicardial activation sequence was represented by a series of isochrones occurring at $5 \mathrm{~ms}$ intervals. These were obtained both during sinus rhythm and ventricular tachycardia.

\section{Results}

\section{Preoperative electrophysiological study (Case} I)

During basic right ventricular driving with a cycle length of $600 \mathrm{~ms}$, single right ventricular premature beats induced with a coupling time ranging from 290 to $330 \mathrm{~ms}$ could initiate the tachycardia. Fig. I is a recording obtained from Case I during tachycardia. The QRS complex shows a right bundlebranch block configuration and there is right axis deviation (mean frontal plane QRS axis from I2 lead electrocardiogram obtained during tachycardia $\left.+115^{\circ}\right)$. No His potential precedes ventricular activation as seen on the His bundle electrogram and atrial activation on the high right atrial electrogram is independent of ventricular activation; these two features strongly suggest the ventricular origin of the tachycardia. The third beat is an induced right ventricular premature beat occurring $290 \mathrm{~ms}$ after the preceding QRS complex. The interval enclosing this ventricular premature beat is $290+330=620$, which is less than twice the basic cycle length of the tachycardia $(2 \times 365=730)$ or less than compensatory. The tachycardia could be terminated by 3 induced right ventricular premature beats with coupling times of 250,180 , and $260 \mathrm{~ms}$. This patient, therefore, has a ventricular tachycardia and the criteria for the diagnosis of an underlying reentry or reciprocal mechanism are fulfilled.

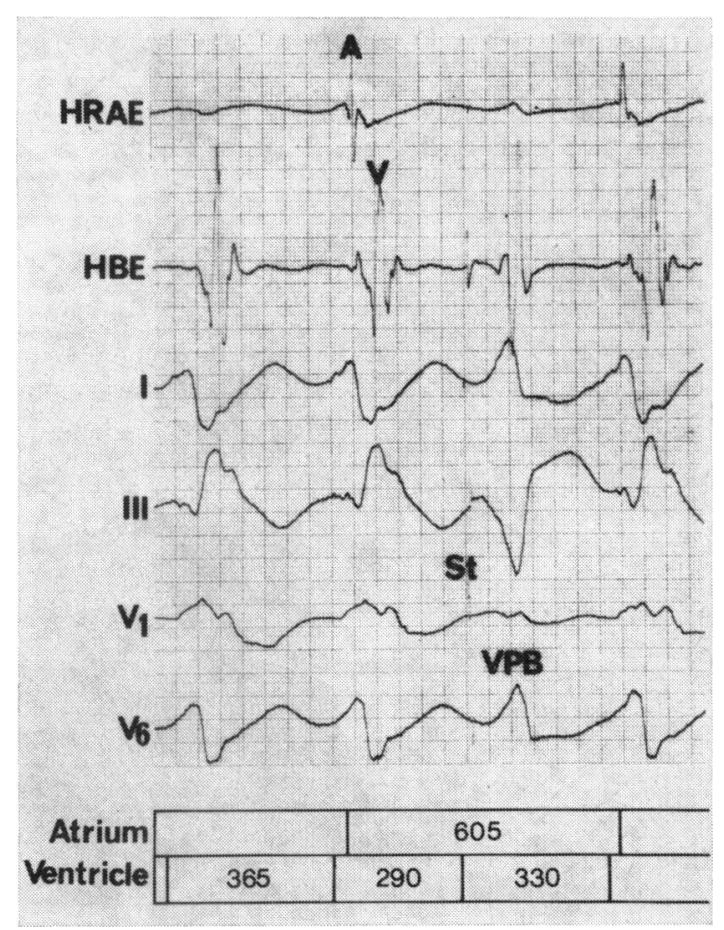

FIG. I Recording obtained from Case I during ventricular tachycardia showing the effect of an induced ventricular premature beat. $H R A E=$ high right atrial electrogram; $H B E=H i$ bundle electrogram; $I$, $I I I, V I, V 6=$ surface electrocardiographic leads; $A=$ atrial potential on the HRAE; $V=$ ventricular potential as seen on the HBE; St=pacemaker stimulus artefact; $V P B=$ ventricular premature beat.

\section{Operative electrophysiological study (Case I)}

Fig. 2 is a semidiagrammatic representation of the left ventricle showing an isochrone map of left ventricular epicardial activation obtained from Case I during sinus rhythm. As can be seen from this figure, there is an extensive area found to be electrically inert, which corresponded to a visible old infarct on the posteroinferior surface of the left ventricle. The sequence of epicardial activation is followed by comparing the specific colours and their appropriate time intervals on the colour chart with the colours as they appear on the drawing of the left ventricle. As can be seen in the figure, the earliest onset of epicardial activation occurs on the anterior surface of the left ventricle adjacent to the distal third of the left anterior descending coronary artery. This earliest point of epicardial activation occurs I 3 ms after the onset of left ventricular cavity potential (see inset of recording). Epicardial activation then occurs in such a manner that after low 

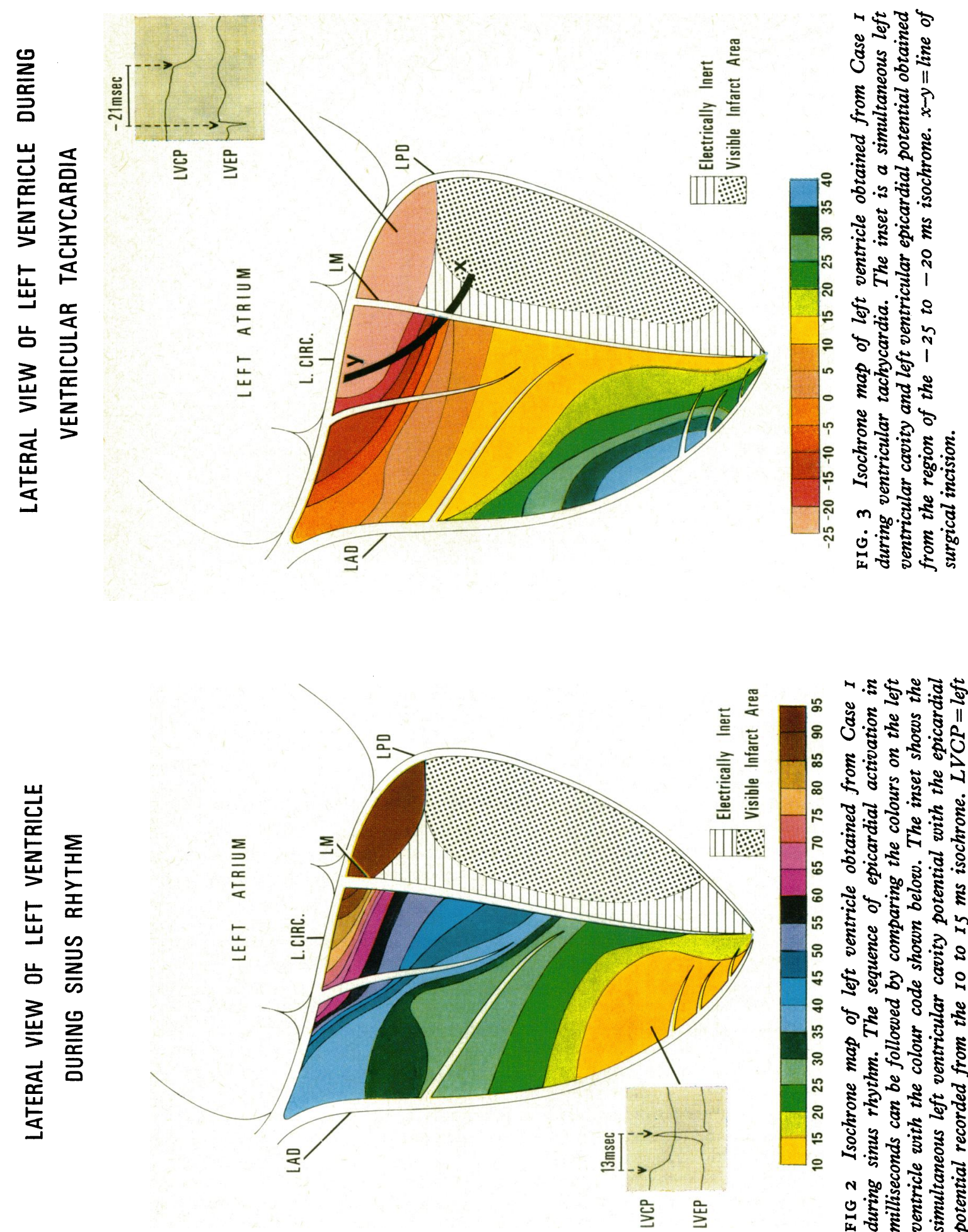

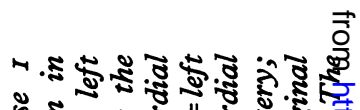

\%ั.

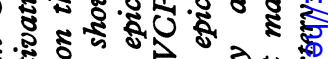

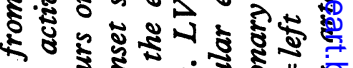

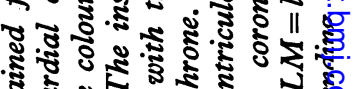

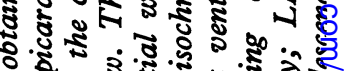

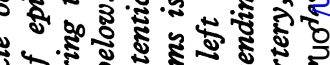

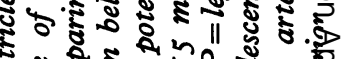
\&

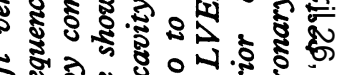

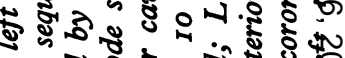
के ค है

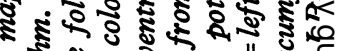

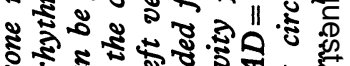

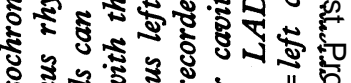

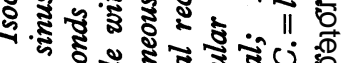
$\sim$ o o : प 
paraseptal activation, epicardial activation spreads to the lateral border of the heart, the upper paraseptal region, and then finally to the posterobasal region of the left ventricle which is the area activated latest at $95 \mathrm{~ms}$ after the onset of the left ventricular cavity potential. It should be noticed that slow conduction is occurring in the lateral and posterobasal regions of the left ventricle, as shown by a much smaller area of ventricle activated between 55 and $95 \mathrm{~ms}$ as opposed to the larger area between ro and $55 \mathrm{~ms}$; this region is adjacent to the upper part of the infarct scar.

Fig. 3 is an isochrone map of left ventricular epicardial activation obtained from Case I during ventricular tachycardia. The colours representing the isochrones are again represented on the colour chart at the bottom of this figure. It will be seen that during ventricular tachycardia the earliest area of epicardial activation is the posterobasal region of the left ventricle above the infarct scar. This area is activated 20 to $25 \mathrm{~ms}$ before the onset of left ventricular cavity potential as is shown on the inset of a left ventricular epicardial potential obtained simultaneously with left ventricular cavity potential from this area. It will be noticed that this area of epicardium is the area in which late and slow activation occurred during sinus rhythm (Fig. 2). After epicardial activation in this region the activation process spreads slowly to the upper paraseptal regions of the left ventricle, and then subsequently the activation process spreads to the apical and lateral regions of the left ventricle. Once again it will be seen that conduction is slow from the posterobasal regions of the left ventricle to the upper paraseptal region ( -25 to $\circ \mathrm{ms}$ conduction time) whereas the time taken for epicardial activation of the whole of the remainder of the left ventricle is 0 to $40 \mathrm{~ms}$, indicating a faster rate of conduction. After epicardial mapping an epicardial to endocardial incision was made across the re-entry front as shown by line $\mathrm{xy}$ in Fig. 3 .

\section{Preoperative electrophysiological study (Case} 2)

During basic right ventricular driving with a cycle length of $600 \mathrm{~ms}$, two right ventricular premature beats with coupling times of 270 and $190 \mathrm{~ms}$, respectively, could initiate the tachycardia. Induced right ventricular premature beats during tachycardia were followed by pauses less than compensatory. The tachycardia could be terminated by two induced right ventricular premature beats with coupling times of $\mathrm{I} 80$ and $\mathrm{I} 70 \mathrm{~ms}$.

Fig. 4 is a recording obtained from Case 2 during tachycardia. The QRS complex shows a

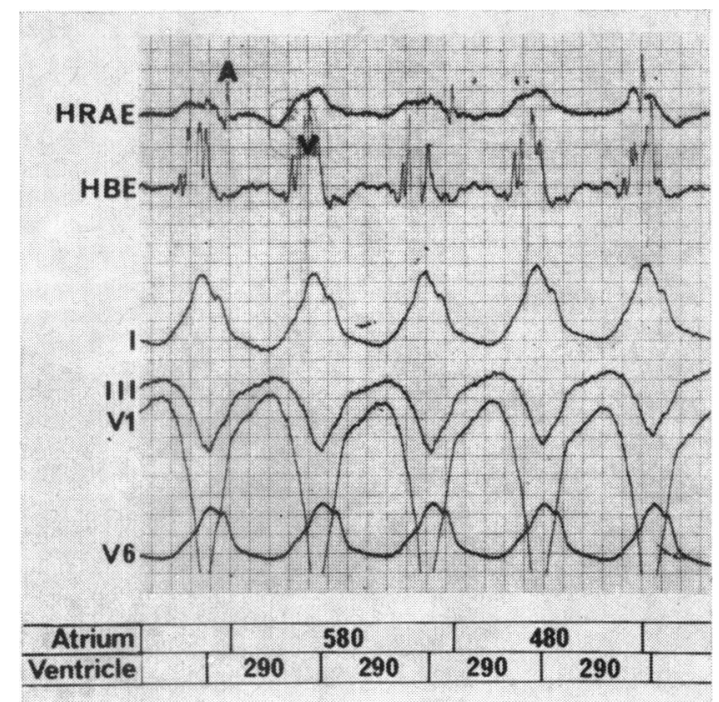

FIG. 4 Recording obtained from Case 2 during ventricular tachycardia.

left bundle-branch block configuration and there is left axis deviation (mean frontal plane QRS axis from the 12 lead electrocardiogram obtained during tachycardia $-30^{\circ}$ ). No His potential precedes ventricular activation as seen on the His bundle electrogram, and atrial activation on the high right atrial electrogram is independent of ventricular activation; these two features strongly suggest the ventricular origin of the tachycardia. The results of this study show this patient to have a ventricular tachycardia and the criteria for the diagnosis of an underlying re-entry or reciprocal mechanism are fulfilled.

\section{Operative electrophysiological study (Case 2)}

Fig. 5 is a semidiagrammatic representation of the right ventricle showing an isochrone map of ventricular epicardial activation obtained from Case 2 during sinus rhythm. As can be seen, the earliest onset of epicardial activation occurs on the anterior surface of the right ventricle adjacent to the anterior intraventricular groove at a site corresponding to the distal third of the anterior descending coronary artery. This area is activated $25 \mathrm{~ms}$ after the onset of the right ventricular cavity potential. Epicardial activation then occurs in such a manner that after activation of the lower right paraseptal region activation spreads to the right anterior and posterior basal regions. The latest point of activation is in the region of the outflow tract of the right ventricle which is activated at $75 \mathrm{~ms}$. In the anterobasal regions of the right ventricle just above the right 

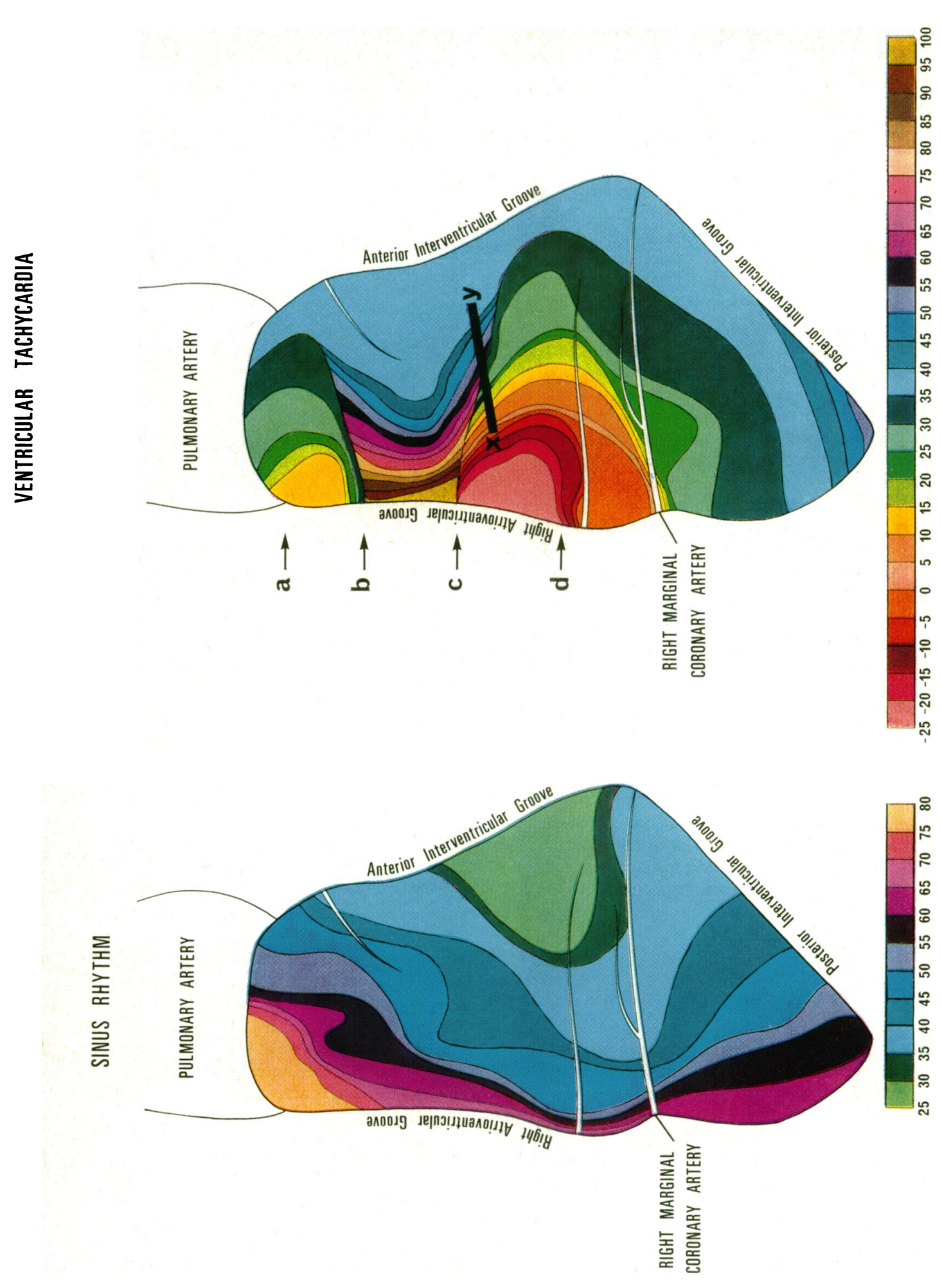
marginal coronary artery rather slower conduction is occurring, this small area being activated between 50 and $70 \mathrm{~ms}$ whereas activation of the whole ventricle up to this point had occurred between 25 and $50 \mathrm{~ms}$.

Fig. 6 is an isochrone map of right ventricular epicardial activation obtained from Case 2 during ventricular tachycardia. It will be seen that during ventricular tachycardia the earliest point of epicardial activation occurred $25 \mathrm{~ms}$ before the onset of right ventricular cavity potential and this area was between points $c$ and $d$ (Fig. 6) on the anterobasal regions of the right ventricle. From this point epicardial activation proceeded towards the anterior interventricular groove and the posterior interventricular groove. In addition there appeared to be a second activation front occurring later at $10 \mathrm{~ms}$ after the onset of right ventricular cavity potential in the region $a$ to $b$ in the region of the outflow tract of the right ventricle. After activation of the anterior and posterior interventricular regions of the right ventricle the activation front appeared to reverse in direction so that the last areas of ventricle to be activated were between $b$ and $c$ on the anterobasal regions of the right ventricle. It will be seen from the tightly packed isochrones that in this region of activation the conduction was slow. It was not possible to join up the isochrones such that the activation fronts were continuous as it was found that the earliest point of activation at $-25 \mathrm{~ms}$ and the latest point of activation at $95 \mathrm{~ms}$ occurred closely adjacent at point $c$, and even using a bipolar electrode with an interelectrode distance of $2 \mathrm{~mm}$ it was not possible to demonstrate a graduation of activation times in this region. The surgeon commented on the presence of scarring in the region of point $c$ on the right ventricle and it is in this region that slow conduction was occurring both during sinus rhythm and ventricular tachycardia.

Fig. 7 is a recording obtained from Case 2 during ventricular tachycardia. The top tracing is a bipolar epicardial recording and it will be seen that for every QRS complex there are two epicardial potentials. The first epicardial potential (EPI) occurs $40 \mathrm{~ms}$ after the onset of right ventricular cavity potential, and this potential was consistently recorded in the region of the 40 to $45 \mathrm{~ms}$ isochrone. The second potential (EP2) was recorded intermittently from this region and other regions within the late activated area of ventricle between $b$ and $c$ and in this instance occurred $175 \mathrm{~ms}$ after the onset of right ventricular cavity potential. The significance of this will be discussed.

After epicardial mapping an epicardial to endocardial incision was made across the re-entry pathway as shown by the line xy in Fig. 6 . In addition the

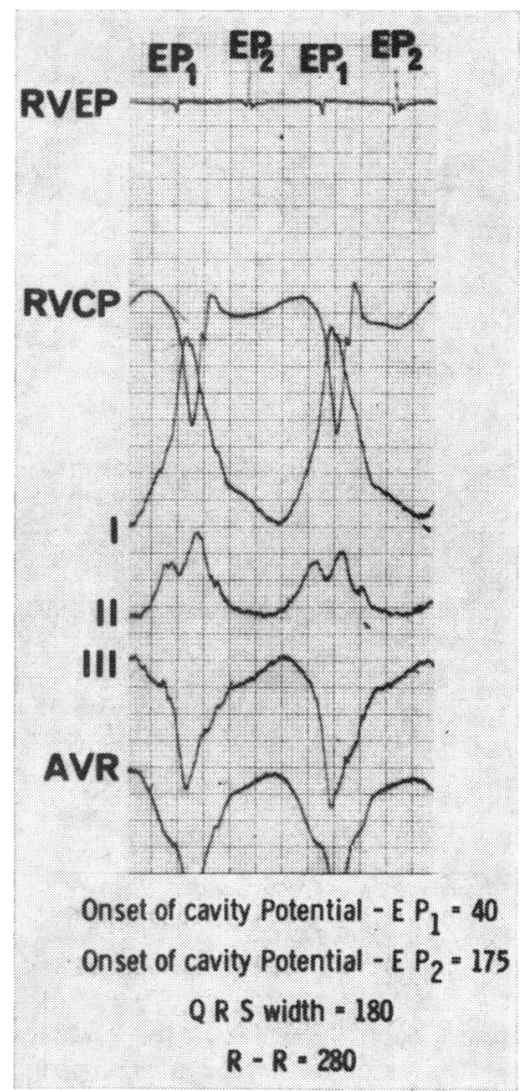

FIG. 7 Simultaneous surface electrocardiogram (leads I, II, III, and AVR), right ventricular cavity potential (RVCP), and right ventricular epicardial potential (RVEP) obtained from Case 2 during ventricular tachycardia from the 40 to $45 \mathrm{~ms}$ isochrone on the right ventricle. EPI=epicardial potential one; $E P_{2}=$ epicardial potential two. The measurements shown below this tracing are in milliseconds.

right bundle-branch was cut. Fig. 8 shows the surface electrocardiographic leads I, II, III, and VI preoperatively (A) and postoperatively (B) after section of the right bundle-branch; note the development of an S wave in lead I, $R$ in III, and an rsR pattern in VI.

\section{Discussion}

\section{Electrophysiological considerations}

A considerable amount of evidence has been accumulated from animal experimental work to support a re-entry or reciprocal process as an underlying mechanism for ventricular premature beats or ventricular tachycardia (Cranefield, Klein, and Hoffman, I97I; Sasyniuk and Mendez, 197I; Wallace 
A

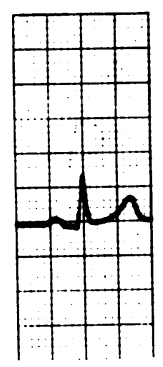

I

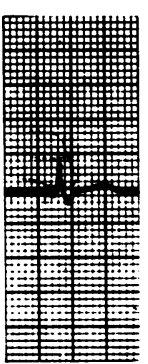

I

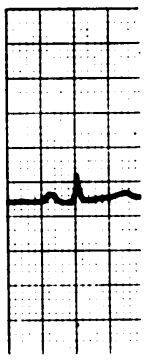

II

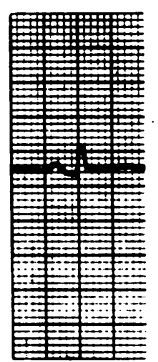

II

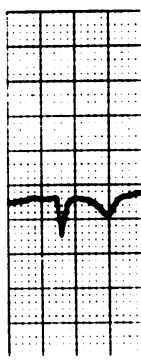

III

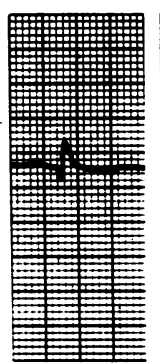

III

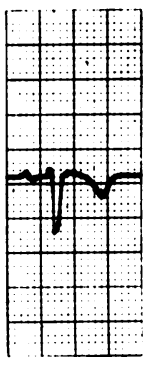

v1

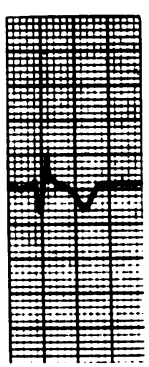

V1
FIG. 8 Surface electrocardiographic recordings of leads I, II, III, and VI obtained from Case 2 preoperatively $(A)$ and postoperatively $(B)$.

and Mignone, 1966; Han, Goel, and Hanson, 1970). More recently, using the techniques of programmed electrical stimulation of the heart it has been shown that a re-entry mechanism may be responsible for ventricular tachycardia in some patients (Wellens et al., 1972) and that the re-entry circuit in patients with re-entry ventricular tachycardia may be in the main intraventricular bundle-branches (Spurrell et al., 1973).

The two patients with paroxysmal ventricular tachycardia presented here have had electrophysiological studies: in each patient the tachycardia could be both initiated and terminated by appropriately timed ventricular premature beats, and induced ventricular premature beats during tachycardia were followed by pauses less than compensatory. This response to premature stimulation is most easily explained if a re-entry process is postulated as the underlying mechanism of the tachycardia. In addition to this study both patients underwent epicardial mapping procedures during ventricular tachycardia in order to obtain more precise localization of the re-entry pathway before surgical interruption of this pathway.

The sequence of epicardial activation during sinus rhythm in both patients was essentially normal (Roos, Van Dam, and Durrer, I968) except that in Case I: the earliest onset of activation at $13 \mathrm{~ms}$ was abnormally early, and no electrical activity was recorded over the posteroinferior surface of the heart due to the old infarct. Both patients, during sinus rhythm, showed areas of slow activation in epicardial regions where the earliest onset of ventricular activation was shown to occur during tachycardia.

Under normal circumstances the onset of left ventricular cavity potential indicates the earliest onset of ventricular endocardial activation (Durrer, Roos, and Van Dam, 1966). In both patients the epicardial activation sequence during tachycardia showed that the earliest onset of ventricular activation occurred between 20 and $25 \mathrm{~ms}$ before the onset of ventricular cavity potential. In Case I the surface electrocardiogram obtained during tachycardia showed a right bundle-branch block with right axis deviation (Table). Previous work from this department has shown that the site of re-entry in ventricular tachycardia may in some instances involve the main intraventricular bundle-branches (Spurrell et al., 1973). If this is so, then in Case I a possible re-entry circuit could involve anterograde conduction by way of the anterior division of the left bundle with retrograde conduction in the posterior division and with block in the right bundle-branch; this would give the electrocardiographic appearances of right bundle-branch block with right axis deviation. The epicardial map allows a more precise analysis of this hypothesis. The earliest point of epicardial activation in Case $I$ is in the posterobasal regions of the left ventricle. By the time epicardial activation is occurring simultaneously with the onset of ventricular cavity potential (earliest onset of left ventricular endocardial activation), the activation front has reached the anterior interventricular groove adjacent to the upper part of the anterior descending coronary artery. Endocardial activation at this site in the upper anterior part of the left side of the interventricular septum is likely to involve activation of the anterior division of the left bundle-branch. Anterograde conduction in the anterior radiation can then occur. This is represented diagramatically in Fig. 9. It will be seen from this Figure that at point $e$ the endocardium is activated simultaneously with the epicardium over it. From point e the impulse would travel anterogradely in the anterior division of the left bundle and then enter the posterior division of the left bundle to pass retrogradely in the region of this division to a point which is just above the infarct scar, where slow conduction has been shown to occur in this patient. It is postulated that in this region the impulse is conducted slowly from the endocardium to the overlying epicardium at point $b$, 


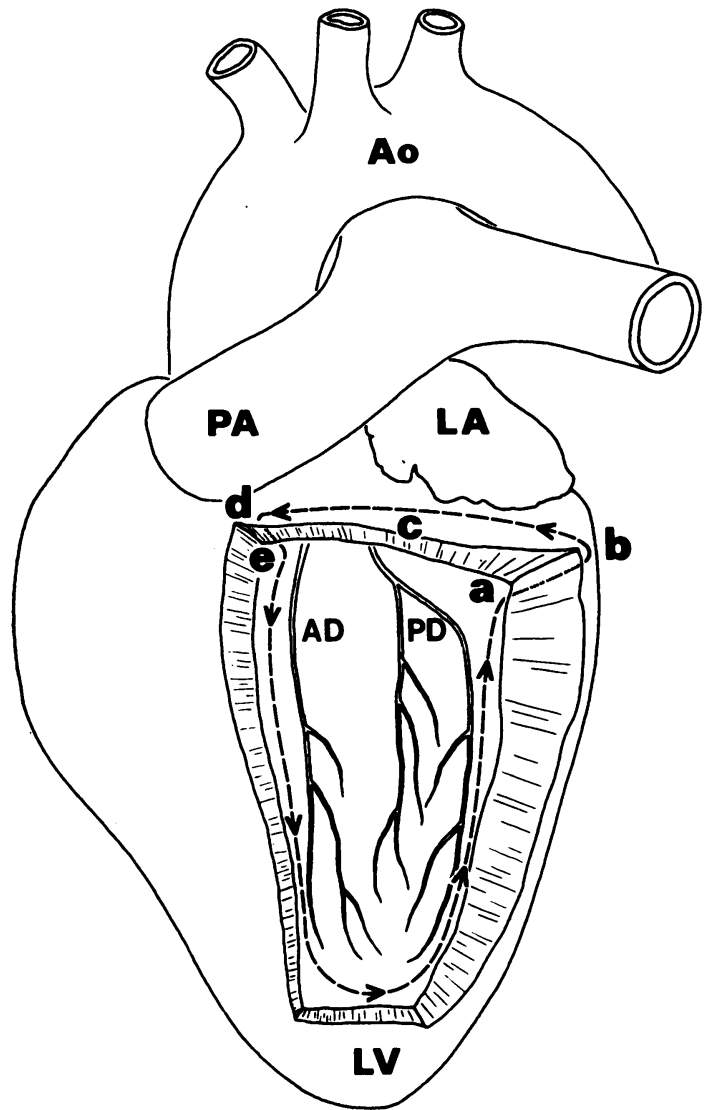

FIG. 9 Drawing of the left ventricle and ventricular cavity to demonstrate the proposed re-entry circuit (interrupted line) in Case I. Ao=aorta; $P A=$ pulmonary artery; $L A=$ left atrium; $L V=$ left ventricle; $A D=$ anterior division of left bundle-branch; $P D=$ posterior division of left bundle-branch; $a, b, c, d, e$ are reference points used in the text.

and it is at this point that the ventricle is reactivated and the process is repeated, thereby sustaining the re-entry. Slow conduction in the region of an ischaemic area as indicated in Case $I$ has been shown experimentally in the dog heart to be an essential part of the re-entry mechanism (Han et al., 1970).

It is this slowing of conduction over a segment of the circuit which allows the tissue ahead, which was depolarized earlier and which is in the path of the activation front, to become non-refractory so that it can be reactivated and thereby sustain the reentry process.

Case 2 showed a QRS configuration of left bundle-branch block with left axis deviation during tachycardia. If the main intraventricular bundle- branches were involved in the re-entry circuit, then this QRS configuration could be explained on the basis of anterograde conduction in the right bundlebranch and retrograde conduction in the region of the anterior division of the left bundle. Examination of the epicardial map obtained from this patient (Fig. 6) shows a complex sequence of activation. Fig. Io is a diagram of the right ventricle showing the postulated re-entry circuit. The earliest point of activation occurs in the region of the anterior atrioventricular groove, at point $a$, and the activation process spreads towards the interventricular groove. In the 0 to $5 \mathrm{~ms}$ isochrone epicardial activation is occurring simultaneously with activation of the endocardium at point $c$, following epicardial to endocardial spread of activation (b to $c$ ). Between $c$ and $d$ the overlying epicardium is known to show slow conduction (points $b$ to $c$, Fig. 6), and it is in this region that slow conduction from endocardium to epicardium occurs (cd to a). Distal to this area of slow conducting tissue in the region of the right outflow tract (a to b, Fig. 6) the ventricle again can conduct more rapidly and this may explain the appearance of a second activation front in this

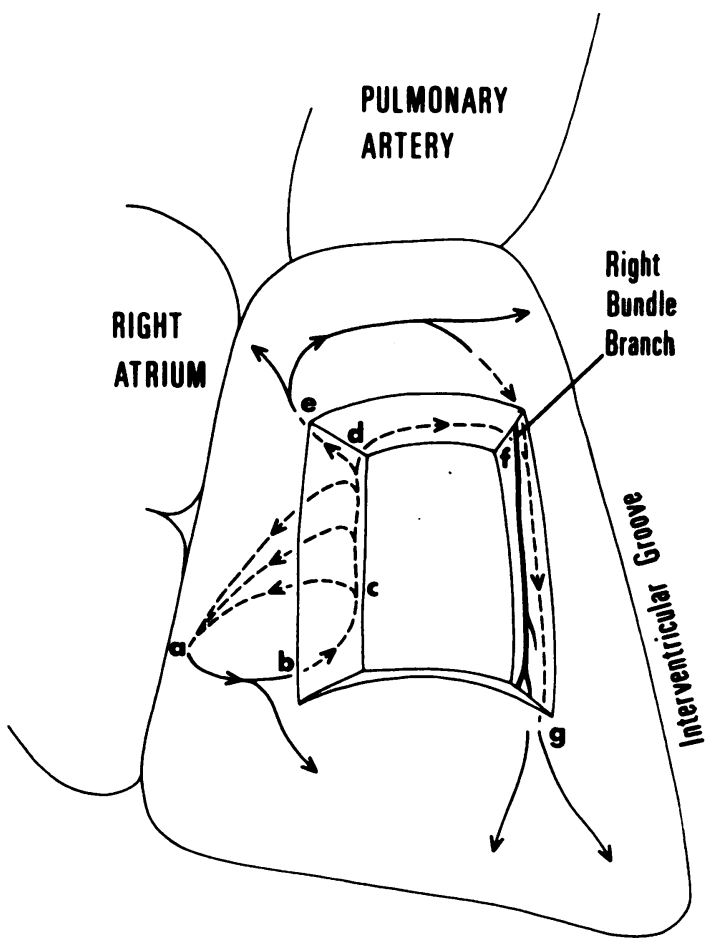

FIG. IO Drawing of the right ventricle and ventricular cavity to show the proposed re-entry circuit (interrupted line) in Case 2. $a, b, c, d, e, f, g$ are reference points used in the text. 
region following rapid endocardial to epicardial activation (d to e, Fig. 10) at a time when slow conduction is occurring in the region of cd to e (Fig. 10). As a result of this, a second area of early epicardial activation occurs at 10 to $15 \mathrm{~ms}$ (a to b, Fig. 6), despite a region of delayed epicardial activation enclosed between the two areas of early activation ( $a$ to $b$ and $c$ to d, Fig. 6). Endocardial activation with appropriate overlying epicardial activation then occurs, and when the impulse has been conducted to the endocardial surface of the right side of the interventricular septum the right bundle-branch is activated (point $f$, Fig. Io). Anterograde conduction then occurs in the right bundle-branch with further epicardial breakthrough at point $g$ (Fig. 10). The result of this activation process is such that the activation front spreads from the right anterior atrioventricular groove towards the anterior and posterior interventricular grooves. Data concerning the activation process of the left ventricle were not obtained because of the limited time available for safe epicardial mapping during tachycardia. This activation process would, therefore, produce the surface electrocardiographic features of left bundlebranch block. After this activation sequence reentry is able to occur as a result of slow conduction occurring from endocardium to epicardium in the region cd to a (Fig. 10). This is the area $b$ to $c$ in Fig. 6 which is known to be activated slowly and this slow conduction allows the ventricular tissue in the region $\mathrm{c}$ to $\mathrm{d}$ (Fig. 6) to become non-refractory following the preceding depolarization so that when the activation front eventually reaches this area, reactivation occurs and re-entry is sustained.

Fig. 7 is a recording of the epicardial potentials recorded from the area of ventricle where slow conduction is occurring. The first potential EPI is thought to represent the true potential recorded from this area. The second epicardial potential EP2 is late and occurs $175 \mathrm{~ms}$ after the onset of cavity potential. EP2 may represent delayed activation within the ventricular wall not necessarily closely adjacent to the recording electrode and result from slow and nonuniform conduction in this region. The findings of slow conduction and greatly delayed epicardial 'after potentials' support the concept that a re-entry mechanism may be the basis for the ventricular tachycardia in this patient.

It should be stressed that the diagrams of the reentry circuits in Fig. 9 and ro have been constructed by consideration of the data obtained from the preoperative electrophysiological studies and the epicardial mapping studies at surgery; these suggested circuits must be speculative, for without simultaneous recordings from numerous sites during tachycardia, particularly from the bundle-branches, it is not possible to determine the re-entry pathway with accuracy and only a relatively crude assessment can be made from the available data.

\section{Approaches to treatment}

Various approaches to the surgical management of refractory ventricular tachycardia have previously been made. Resection of a ventricular aneurysm is well recognized in the management of ventricular tachycardia (Thind, Blakemore, and Zinsser, 197I), and resection of an aneurysm or a hypokinetic area of left ventricular wall with additional aortocoronary bypass surgery has also been shown to be successful in the treatment of this arrhythmia (Graham et al., 1973). Nitter-Hauge and Storstein (1973) found that sympathectomy abolished abnormal ventricular activity in 2 out of 5 patients with ventricular tachycardia, and recurrent ventricular tachycardia was abolished in 7 out of 12 patients who underwent resection of either a ventricular aneurysm or an akinetic area of the left ventricular wall.

Surgical interruption of part of the re-entry circuit has been shown to be successful in the management of paroxysmal tachycardia in patients with the Wolff-Parkinson-White syndrome (Cobb et al., 1968). A similar principle can be applied to patients with re-entry ventricular tachycardia and it may be that removal of an aneurysm or an akinetic area of ventricular wall is successful in abolishing attacks of ventricular tachycardia because it involves excision of part of the re-entry circuit. Spurrell et al. (1973) described 2 patients with re-entry ventricular tachycardia in whom the anterior division of the left bundle-branch was thought to constitute the anterograde pathway of the reciprocal circuit and in both patients the anterior division was interrupted surgically; one patient succumbed after an additional left ventricular aneurysm resection but the other patient survived, is well, and free from tachycardia. Fontaine et al. (1973) have also successfully interrupted the re-entry circuit surgically in ventricular tachycardia.

The procedure of epicardial mapping requires the maximum practical exposure of the heart, and an approach using a sternal splitting incision best provides this. The heart has to be raised to allow adequate epicardial mapping of the diaphragmatic surface of the right ventricle and posterior surface of the left ventricle. The procedure necessitates close attention to the details of the mapping while at the same time constantly maintaining a satisfactory cardiovascular state; this is especially relevant when the mapping is being performed during tachycardia.

It is important initially to draw an accurate semidiagrammatic map of the ventricular surfaces. All 
landmarks on the ventricles, e.g. coronary vessel branches, fixed epicardial creases, epicardial fibrotic patches, or myocardial scars, etc. are accurately entered on the diagram. This must be done by the surgeon at the operation in order to identify the exact site of the epicardial potentials on the heart's surface.

As each record is taken the epicardial activation times are measured so that only a few minutes are required, after completion of the mapping, to analyse the information. After completion of the isochrone map a myotomy incision to interrupt any apparent re-entry circuit can be planned.

When the desired incision has been accurately located on the diagram it is then possible, because of the noted anatomical landmarks, to translate this to the heart itself. By the use of a series of atraumatic stainless steel sling sutures which penetrate the whole ventricular wall thickness and lie across the line of the proposed incision, it is possible to incise completely through the myocardium and control bleeding by gentle cross tension on the steel sutures. The incision can then be formally closed by a usual myocardial suture technique.

Should a coronary artery be closely related to or cross the proposed line of incision, the involved artery should be mobilized sufficiently so that the incision can be made without cutting the artery or endangering the artery lumen during subsequent suturing of the ventriculotomy.

It appears important that the incision extends completely through the ventricular wall to ensure that no minor myocardial bridge, and therefore potential conduction pathway, remains. If, as in Case I, such a pathway extends up to the left atrioventricular ring, it is not possible safely to extend the incision into the mitral ring. In this case it is necessary to leave a small muscular remnant undivided to ensure competent closure of the incision without risk of damage to the mitral valve apparatus.

It appears likely that the majority of these cases can be operatively treated without resorting to cardiopulmonary bypass, but it is necessary to have this facility immediately available.

The site of re-entry in the 2 patients described here was assessed using epicardial mapping techniques, as detailed above, and in both patients an epicardial to endocardial incision was made across the re-entry front (x-y, Fig. 3 and 6). In Case I the incision was limited to cutting across the anterograde part of the re-entry circuit only (x-y, Fig. 3). In Case 2, however, the right bundle-branch was cut in addition, because the re-entry circuit was found to be complex at surgery and it was, therefore, felt advisable to interrupt the re-entry circuit at two different points; it will be seen from the position of the incision in Fig. 6 that both the anterograde and slow conducting retrograde parts of the re-entry circuit have been divided by the incision in this patient.

Case I had a total of only three attacks of tachycardia in the immediate 2 months after operation, and in the following 5 months, up to the time of writing, there have been no further attacks. The occurrence of these early attacks may be related to the fact that it was not possible totally to interrupt the re-entry front because the incision could not be extended up to the atrioventricular groove (see Fig. 6) and in this region conduction could, therefore, still occur. However, the patient has been improved dramatically by contrast to his perilous preoperative condition. Case 2 has had no attacks of tachycardia postoperatively (4 months to the time of writing) and now leads a normal life.

This approach to the surgical management of ventricular tachycardia demonstrates a technique by which major cardiac surgery can be avoided. In Case I the incision could be made without the use of cardiopulmonary bypass and without the need for resection of a large area of ventricular wall. In Case 2 there was no evidence of a ventricular aneurysm or coronary artery disease so that the usually accepted surgical therapy for ventricular tachycardia could not be carried out and it was felt that the only effective surgical treatment available was interruption of the re-entry circuit. A short period of cardiopulmonary bypass was used in this patient in order to cut the right bundle-branch, but as mentioned above, an epicardial to endocardial incision appropriately placed might have been all that was required to successfully interrupt the reentry circuit.

This surgical approach to the management of refractory, life-threatening ventricular tachycardia provides the possibility of successful therapy without having to use cardiopulmonary bypass and without the need for procedures involving resection of large areas of ventricular wall with or without additional aortocoronary bypass. In patients with ventricular tachycardia in whom the aetiology is not coronary artery disease the surgical approach described above may be the only one available in the management of this life-threatening arrhythmia. It also has the attraction of being logically based on electrophysiological observations made directly on the patient before and at the time of operation.

This work was supported by a British Heart Foundation Grant, and acknowledgements are made to Mr. E. Boyd (Physics Department, Guy's Hospital) and to Devices Instruments Ltd. for technical assistance. 
The authors are grateful to Dr. R. Gibson for allowing them to publish the details of the patient referred from the Brompton Hospital.

\section{References}

Cobb, F. R., Blumenschein, S. D., Sealy, W. C., Boineau, J. P., Wagner, G. S., and Wallace, A. G. (1968). Successful surgical interruption of the bundle of Kent in a patient with Wolff-Parkinson-White syndrome. Circulation, 38, ror 8.

Cranefield, P. F., Klein, H. O., and Hoffman, B. F. (197I). Conduction of the cardiac impulse: I. Delay, block, and one-way block in depressed Purkinje fibres. Circulation Research, 28, 199.

Durrer, D., Roos, J. P., and Van Dam, R.Th. (1966). The genesis of the electrocardiogram of patients with ostium primum defects (ventral atrial septal defects). American Heart fournal, 71, 642.

Fontaine, G., Frank, R., Bonnet, M., Cabrol, C., and Guiraudon, G. (1973). Méthode d'étude expérimentale et clinique des syndromes de Wolff-Parkinson-White et d'ischémie myocardique par cartographie de la dépolarisation ventriculaire épicardiaque. Coeur et Médecine Interne, 12, 105.

Graham, A. F., Craig Miller, D., Stinson, E. B., Daily, P. O., Fogarty, T. J., and Harrison, D. C. (1973). Surgical treatment of refractory life-threatening ventricular tachycardia. American fournal of Cardiology, 32, 909.

Han, J., Goel, B. J., and Hanson, C. S. (1970). Re-entrant beats induced in the ventricle during coronary occlusion. American Heart fournal, 80, 778.

Lown, B., Kleiger, R., and Williams, J. (1965). Cardioversion and digitalis drugs: changed threshold to electric shock in digitalized animals. Circulation Research, 17, 519.

Nitter-Hauge, S., and Storstein, O. (1973). Surgical treatment of recurrent ventricular tachycardia. British Heart fournal, 35, II32.

Roos, J. P., Dan Dam, R.Th., and Durrer, D. (1968). Epicardial and intramural excitation of normal heart in six patients 50 years of age and older. British Heart fournal, 30, 630 .
Sasyniuk, B. I., and Mendez, C. (I97I). A mechanism for re-entry in canine ventricular tissue. Circulation Research, $28,3$.

Scherlag, B. J., Lau, S. H., Helfant, R. H., Berkowitz, W. D., Stein, E., and Damato, A. N. (1969). Catheter technique for recording His bundle activity in man. Circulation, 39, I3.

Spurrell, R. A. J., Sowton, E., and Deuchar, D. C. (1973). Ventricular tachycardia in four patients evaluated by programmed electrical stimulation of the heart and treated in two patients by surgical division of anterior radiation of left bundle-branch. British Heart fournal, 35, ror4.

Thind, G. S., Blakemore, W. S., and Zinsser, H. F. (197I). Ventricular aneurysmectomy for the treatment of recurrent ventricular tachyarrhythmia. American fournal of Cardiology, 27, 690.

Wallace, A. G., and Mignone, R. J. (1966). Physiologic evidence concerning the re-entry hypothesis for ectopic beats. American Heart fournal, 72, 60.

Wellens, H. J. J., Schuilenburg, R. M., and Durrer, D. (1972). Electrical stimulation of the heart in patients with ventricular tachycardia. Circulation, 46, 216.

\section{Addendum}

Since submission of this manuscript for publication, two further patients with life-threatening re-entry ventricular tachycardia have undergone epicardial mapping at cardiac surgery. In one patient the re-entry circuit was found in the outflow tract of the right ventricle involving the adjacent interventricular septum, and an incision was made across the re-entry front in this region. In the other patient the re-entry circuit was found in the left ventricle involving the middle third of the interventricular septum, and an appropriate incision was made across the re-entry front at this site. Both patients are now totally free from attacks of tachycardia.

Requests for reprints to Dr. R. A. J. Spurrell, Cardiac Department, St. Bartholomew's Hospital, London E.C.I. 